\title{
Incorporação de fibroina de Bombyx mori e nanotubos em sistemas de poliamida 6
}

\author{
Paulo Rodrigo Stival Bittencourt*, Luciano Moura de Souza, Natália Zanotelli, Fernando \\ Reinoldo Scremin

\begin{abstract}
Laboratório de Análises Térmicas e Espectrometria de Materiais e Combustíveis, Universidade Tecnológica Federal do Paraná,
\end{abstract} \\ Medianeira, 85884-000, Paraná, Brasil. *paulob@utfpr.edu.br
}

Recebido: 11 julho 2018 / Aceito: 10 setembro 2018 / Publicado online: 12 setembro 2018

\begin{abstract}
Resumo
A fibroina da seda é um biopolímero natural proveniente de Bombyx mori L. (bicho da seda) que possui excelente estabilidade térmica e resistência mecânica. Parte deste biopolimero é rejeitada no processo industrial devido a escolha de casulos viáveis para a produção de seda de qualidade. O objetivo dessa pesquisa foi reaproveitar o biopolímero natural proveniente de $B$. mori para produzir sistemas, processados por casting, de misturas de poliamida $6 \mathrm{com}$ nanotubo de carbono funcionalizado com carboxila e a fibroina. O filme polimérico foi obtido por casting, utilizando ácido fórmico como solvente. As propriedades físico-químicas foram analisadas por Espectroscopia no Infravermelho, análise termogravimétrica e ensaios de tração mecânica. Os resultados das análises reportaram o aumento na estabilidade térmica e mecãnica dos filmes com a presença de fibroina e MWNT nos compósitos de poliamida 6, tendo atingido um módulo de elasticidade de 4,0 GPa com 0,025\% em massa de nanotubo no compósito. Assim, a incorporação da fibroína, extraída de casulos considerados defeituosos, em compósito com poliamida 6 e nanotubo de carbono funcionalizado com carboxila melhorou a estabilidade mecânica nestes sistemas quando comparado a poliamida 6 pura, formando um sistema com potencial uso como material de engenharia.
\end{abstract}

Palavras-chave: bicho da seda, compósitos poliméricos, carbono.

\section{Incorporation of fibroin from Bombyx mori and nanotubes in polyamide 6 systems}

\begin{abstract}
Silk fibroin is a natural biopolymer from Bombyx mori (silkworm) that has excellent thermal stability and mechanical strength. Amounts of this biopolymer is rejected in the industrial process due to the choice of viable cocoons for the production of superior quality silk. This research aimed to reuse the natural biopolymer from B. mori to produce systems, melt processes, polyamide 6 mixtures with carbon nanotube (MWNT) functionalized with carboxyl and fibroin. The polymeric film was obtained by casting using formic acid as the solvent. The results show an increase in the mechanical stability of the films with the presence of fibroin and MWNT in the nylon 6 composites, having reached a modulus of elasticity of $4.0 \mathrm{GPa}$ with $0.025 \%$ of nanotube in the composite. Thus, the incorporation of fibroin, extracted from cocoons considered defective, in composite with polyamide 6 and carbon nanotube functionalized with carboxyl, which the aim was improve the mechanical stability in these systems.
\end{abstract}

Keywords: silkworm, polymer composites, carbon.

\section{Introdução}

$\mathrm{O}$ uso dos produtos naturais em substituição aos sintéticos, derivados de petróleo, tem incentivado as indústrias a pesquisar formas de produção menos nocivas ao meio ambiente, seja no processo produtivo ou na utilização de matérias primas.

O aproveitamento de resíduos da agroindústria é uma forma de valorizar economicamente subprodutos gerados no processo de produção, gerando um ciclo de vida a mais no material descartado. Algumas indústrias perdem parte da matéria prima na etapa de produção por não possuir qualidade necessária, como a indústria de produção de fios de seda que gera uma quantidade significativa de resíduos em todo processo produtivo, na forma de casulos inviáveis para a fiação de seda, alcançando até $10 \%$ em massa do matéria prima, um quantidade expressiva devido ao processamento de seda no Brasil que, em 2016 chegou a 2,2 mil toneladas (Oliveira et al., 2017; Valentini et al., 2018).

Os casulos de B. mori (bicho da seda) defeituosos, denominados residuais, podem ser reaproveitados pois são materiais com excelentes propriedades físico-químicas e podem ser utilizados em misturas poliméricas, por exemplo, na produção de materiais diversos devido à sua compatibilidade biológica e biodegradabilidade (Lopes et al., 2016).

A fibroina é um componente do fio da seda que confere propriedades mecânicas necessárias para que esse produto seja 
utilizado como material de engenharia avançada, principalmente quando aplicado em sistemas de blendas e/ou compósitos com polímeros que possuam boa interação intermolecular, como as poliamidas (Koh et al., 2015).

As Poliamidas possuem grande aplicabilidade comercial para diversos fins por possuir excelentes propriedades físicoquímicas, alguns trabalhos reportam que a inserção de nanotubo de carbono, formando sistemas compósitos, tendem a melhorar estas propriedades e, consequentemente, o desempenho do produto final.(Alef et al., 2018; Guo et al., 2018; Takeuchi et al., 2018; Baek et al., 2017).

Essa pesquisa objetivou produzir sistemas, processados por casting, de misturas de poliamida 6 com nanotubo de carbono (MWNT) funcionalizado com carboxila e fibroina, obtida da seda do casulo de Bombyx mori, obtendo-se filmes finos de compósitos com propriedades térmicas e mecânicas diferenciadas da poliamida 6 pura.

\section{Material e Métodos}

Para a obtenção da fibroína (FS), os casulos de B. mori, cedidos pela BRATAC, fiação de seda de Londrina-PR, foram cortados e imersos em água destilada na proporção 4:1000 (m:v) e aquecidos a $120^{\circ} \mathrm{C}$ por 1 hora em autoclave, para remover sericina que recobre a seda, e submetidos a secagem em estufa a $60{ }^{\circ} \mathrm{C}$ por $7 \mathrm{~h}$. Foi preparada uma solução de FS em ácido fórmico de $20 \mathrm{~g} \mathrm{~L}^{-1}$. A poliamida 6 peletizada (PA6, Sigma Aldrich - CAS 25038-54-4) foi solubilizado em ácido fórmico em concentração de $20 \mathrm{~g} \mathrm{~L}^{-1}$. Os nanotubos utilizados eram de multicamada (Multi-Walled Carbon Nanotube - MWNT) funcionalizados com carboxila, Marca Sigma Aldrich, média de diâmetro de 9,5 nm e largura de $1,5 \mu \mathrm{m}$, com os MWNT foi preparada uma suspensão em ácido fórmico de $1 \%$ em massa.

As soluções de FS, PA6 foram misturadas em proporções para produzir um filme de $50 \%$, em massa, de ambos polimeros, sendo vertidos $10 \mathrm{~mL}$. Dessa mistura em placas de petri de teflon, também foi inserido determinado volume da suspensão de MWNT para produzir um filme de FS/PA6 com $0,025 \%$ do nanotubo em massa. Os filmes de FS/PA6 e FS/PA6/MWNT foram obtidos pelo processo de casting.

Os filmes foram caracterizados por análise de espectroscopia na região do infravermelho (FTIR) usando um espectrômetro PerkinElmer modelo Frontier, no modo de Refletância total atenuada (ATR), com resolução de $4 \mathrm{~cm}^{-1}$ na região entre $4000 \mathrm{~cm}^{-1}$ a $600 \mathrm{~cm}^{-1}$. A análise termogavimetria (TGA) e a foi realizada com analisador térmico stimultâneo STA6000-PerkinElmer em atmosfera inerte de Nitrogênio $\left(\mathrm{N}_{2}\right)$, fluxo de gás a $20 \mathrm{~mL} \mathrm{~min}{ }^{-1}$, intervalo de temperatura entre $70{ }^{\circ} \mathrm{C}$ a $650{ }^{\circ} \mathrm{C}$, com razão de aquecimento em $10^{\circ} \mathrm{C}$ $\min ^{-1}$, foi utilizado porta amostras de platina com aproximadamente $6 \mathrm{~g}$ de amostra.

Os ensaios de tração foram realizados em texturômetro (modelo TA.HD - Plus Stable Micro Systems).Os filmes foram recortados ( 1,0 x 4,0 cm de cada amostra) e avaliados utilizando célula de carga de $5 \mathrm{~kg}$, velocidade de tração $1 \mathrm{~mm}$ $\mathrm{min}^{-1}$, temperatura de ensaio a $25{ }^{\circ} \mathrm{C}$, com os grampos dispostos a 2,54 cm (1 polegada) entre ambos, utilizando o método de teste padrão para propriedades de tração de filmes finos adaptada (ASTM D882-02; Moraes et al., 2016).

\section{Resultados e Discussão}

Na Figura 1 são observadas a caracterização térmica obtida por análise termogravimétrica e a primeira derivada da curva TG (dTG) dos polímeros puros PA6 e FS, ambos apresentam uma única etapa de perda de massa pronunciada, no entanto observa-se que PA6 tem maior estabilidade térmica quando comparado a FS, cuja temperatura de degradação inicial ( $\left.\mathrm{T}_{\text {onset }}\right)$ para FS é de $287^{\circ} \mathrm{C}$, atingindo seu pico de degradação em 320 ${ }^{\circ} \mathrm{C}$, em PA6 a $\mathrm{T}_{\text {onset }}$ é de $390{ }^{\circ} \mathrm{C}$, e o pico de degradação em $430{ }^{\circ} \mathrm{C}$, FS teve uma massa residual final próxima a $33 \%$, enquanto PA6 foi de $2 \%$.

Os espectros FTIR de FS e PA6 mostram as bandas caraterísticas para FS (Figura 2), relativas as amidas I (estiramento $\mathrm{C}=\mathrm{O}, 1620 \mathrm{~cm}^{-1}$ ), amidas II (deformação $\mathrm{N}-\mathrm{H}$, $1510 \mathrm{~cm}^{-1}$ ) e amida III (estiramento C-N, $1228 \mathrm{~cm}^{-1}$ ). A banda na amida I pode ser usado para determinar a conformação molecular, a região entre $1616-1637 \mathrm{~cm}^{-1}$ e $1658-1677 \mathrm{~cm}^{-1}$ é atribuída a conformação de folha- $\beta$ (Chen et al. 2001). Para PA6, a banda em $1725 \mathrm{~cm}^{-1}$ é atribuída especificamente ao grupo $\mathrm{C}=\mathrm{O}$, em $1635 \mathrm{~cm}^{-1}$ corresponde a deformação axial amida, em $1538 \mathrm{~cm}^{-1}$ são atribuídas especificamente a amidas secundárias e em $3300 \mathrm{~cm}^{-1}$ observa-se a banda característica da do estiramento da ligação N-H.

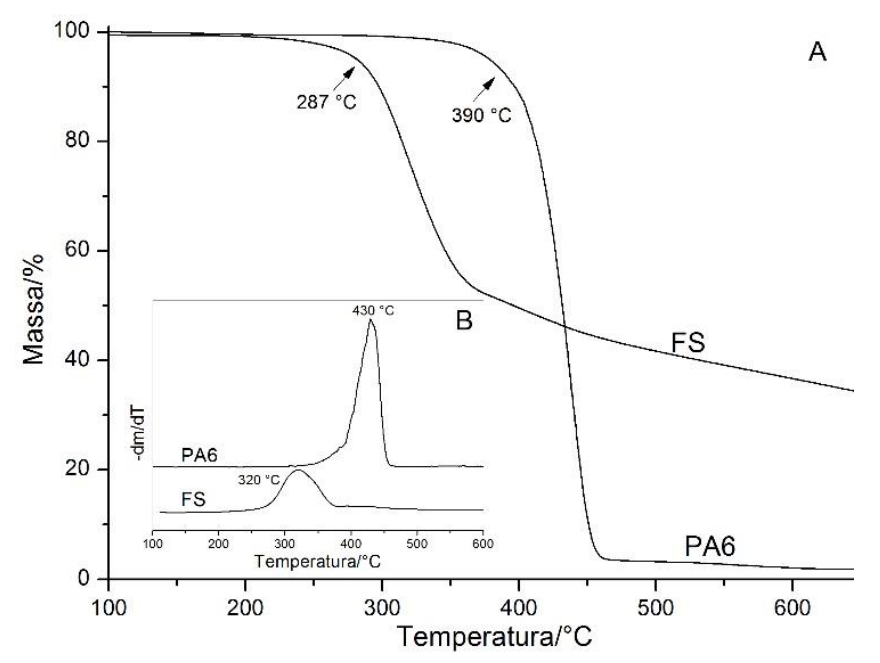

Figura 1. Curva termogravimétrica - TG (A) a primeira derivada da curva TG - dTG; -dm/dT (B) para as amostras poliamida 6 (PA6) e fibroina de seda (FS).

No espectro FTIR da mistura FS/PA6, houve a similaridade das bandas deste sistema com as do PA6 puro, mas este sistema tinha uma conformação cristalina maior entre as cadeias, pois observou-se um aumento de intensidade da banda entre $1658 \mathrm{e}$ $1677 \mathrm{~cm}^{-1}$. Apesar da similaridade entre os espectros de PA6 e FS/PA6, as bandas referentes a amida I e II nestes filmes, tem perfis similares ao espectro da fibroina, o que pode indicar forte interação entre os polímeros. O espectro FTIR do sistema FS/PA6/MWNT mostra que a inserção dos nanotubos no sistema ocorrem com a interação deste com os polímeros, segregando e expondo a fibroina na superfície do filme, no entanto a conformação de folha- $\beta$ continua. $O$ aparecimento de uma banda estreita no espectro de FS/PA6/MWNT, em 1670 $\mathrm{cm}^{-1}$, ocorre devido a presença de $\mathrm{C}=\mathrm{O}$ conjugada dos grupos carboxilicos do MWNT, este sinal deveria estar próximo a 
$1680 \mathrm{~cm}^{-1}$, o deslocamento desta banda indica que ocorre interação do nanotubo com os polímeros.

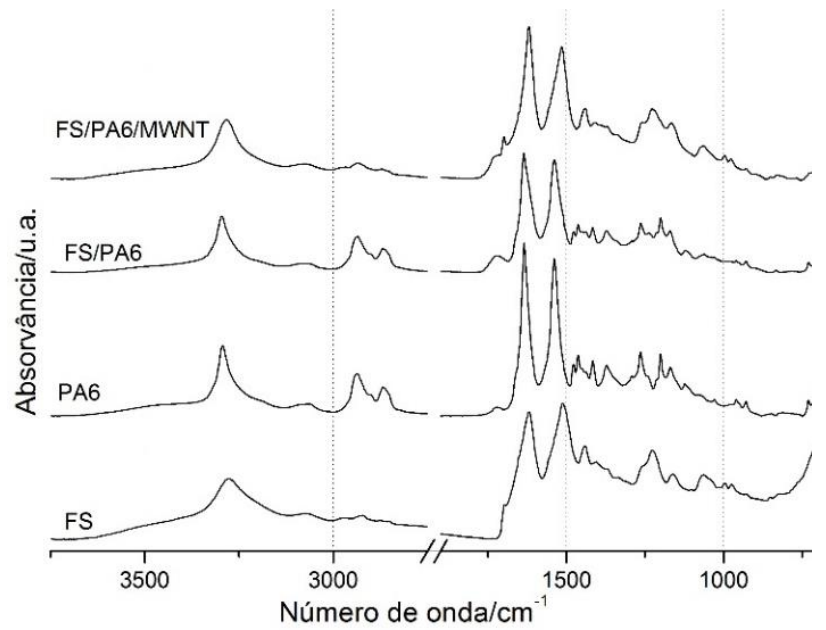

Figura 2. Espectros FTIR de sistemas de poliamida 6 pura (PA6), fibroina de seda (FS), PA6/FS e FS/PA6/Nanotubo (MWNT).

As curvas termogravimétricas das misturas com e sem nanotubos (Figura 3A) tiveram perfis similares, no entanto os sistemas contendo MWNT tiveram sua estabilidade térmica melhorada, deslocando o início da etapa principal de degradação para uma maior temperatura, este efeito é causado devido a interação entre os nanotubos e a matriz polimérica. $\mathrm{Na}$ Figura 3B é possível perceber que na dTG das misturas contendo nanotubo as curvas de degradação térmica têm as etapas mais definidas, comprovando a segregação de fases.

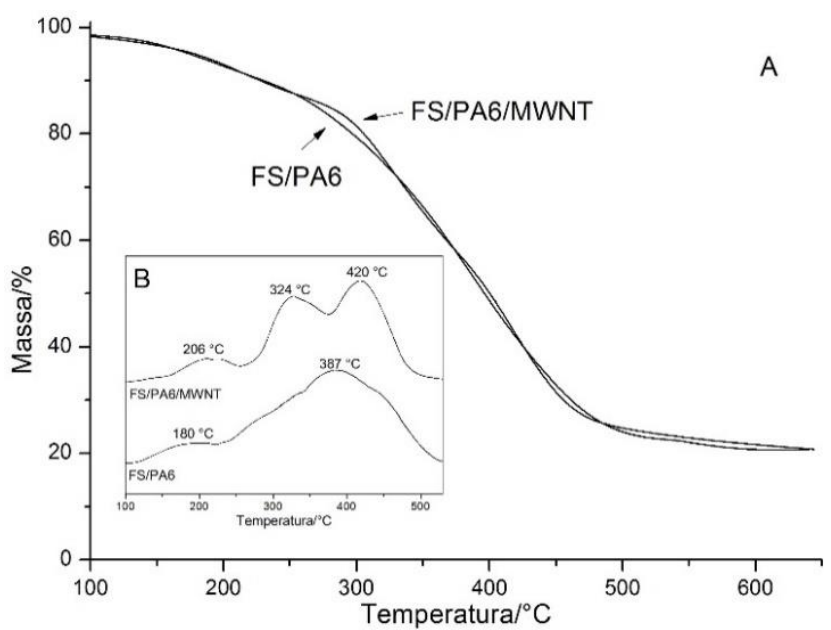

Figura 3. Curva termogravimétrica - TG (A) e dTG (B) para as amostras de poliamida 6 (PA6) e fibroina de seda (FS/PA6) e esta combinação com o nanotubo de carbono (FS/PA6/MWNT).

Os ensaios de tração mostram que os sistemas contendo MWNT tiveram a resistência a tração e módulo de elasticidade aumentados quando comparados ao sistema sem nanotubo, 37,5 \pm 2,0 $\mathrm{MPa}$ e 4,0 \pm 0,3 GPa, respectivamente, para FS/PA6; 49,1 \pm 3,7 MPa e 4,2 \pm 0,4 GPa para
FS/PA6/MWNT, comprovando que, apesar da segregação de fases no sistema, o MWNT reforçou mecanicamente os filmes devido a interações químicas dos nanotubos com a matriz polimérica, o valor obtido para o módulo de elasticidade das amostras é superior ao de filmes finos de poliamida 6 pura, cujo valor varia entre 1,9 a 2,7 GPa (Lv et al., 2015).

\section{Conclusões}

O uso da fibroína em sistemas poliméricos com PA6 demostram ser um bom material para incorporação no sistema e reutilização e valorização dos casulos de $B$. mori descartados, pois ocorre interação entre FS e o PA6. A incorporação do MWNT na blenda como agente de reforço, mesmo que haja certa segregação de fase entre os polímeros, é uma proposta para utilização do nanotubo funcionalizado no processamento destes sistemas, formando materiais mecanicamente reforçados cuja estabilidade térmica e mecânica se mostra superior ao da poliamida 6 pura.

\section{Referências}

Alef, A.; Pereira, C.; Rosenberg, R.; Roberto, J. 2018. Effects of incorporating titanate nanotubes on the mechanical properties of polyamide 11. Polymer Testing, 68: 238-247.

ASTM, D882-02. Standard Test Method for Tensile Properties of Thin Plastic Sheeting.

Baek, Y.; Joong, H.; Kim, S.; Lee, J.; Yoon, J. 2017. Evaluation of carbon nanotube-polyamide thin-film nanocomposite reverse osmosis membrane: Surface properties, performance characteristics and fouling behavior. Journal of Industrial and Engineering Chemistry, 56(dez.): 327-334.

Chen, X.; Knight, D. P.; Shao, Z.; Vollrath, F. 2001. Regenerated Bombyx silk solutions studied with rheometry and FTIR. Polymer, 42(25): 9969-9974.

Guo, Y.; Mi, Y.; Zhao, F.; Ji, Y.; An, Q.; Gao, C. 2018. Zwitterions functionalized multi-walled carbon nanotubes/polyamide hybrid nanofiltration membranes for monovalent/divalent salts separation. Separation and Purification Technology, 206(nov.): 59-68.

Koh, L.; Cheng, Y.; Teng, C.; Khin, Y.; Loh, X.; Tee, S.; Han, M. 2015. Structures, mechanical properties and applications of silk fibroin materials. Progress in Polymer Science, 46(jul.): 86-110.

Lopes, T.; Carvalho, A.; Gurgel, M.; Vieira, A.; Luis, M.; Gurgel, M. 2016. Biosorption study of copper and zinc by particles produced from silk sericine alginate blend : evaluation of blend proportion and thermal crosslinking process in particles production. Journal of Cleaner Production, 137(nov.), 1470-1478

Lv, F.; Yao, D.; Wang, Y.; Wang, C.; Zhu, P. 2015. Recycling of waste nylon 6/spandex blended fabrics by melt processing. Composites Part B, 77(ago.): 232-237.

Moraes, T.; Maria, T.; Costa, H.; Oliveira, A. De, Hickmann, S. 2016. Valorization of food-grade industrial waste in the obtaining active biodegradable films for packaging. Industrial Crops \& Products, 87(set.): 218-228.

Oliveira, R. A.; Santos, J. A.; Boroviecz, S. 2017. Análise do custo de produção e do processo produtivo da sericicultura : um estudo de caso no Paraná. Paraná, Redes - Revista do Desenvolvimento Regional, 22(1): 528-555.

Takeuchi, K.; Takizawa, Y.; Kitazawa, H.; Fujii, M.; Hosaka, K. 2018. Salt rejection behavior of carbon nanotube-polyamide nanocomposite reverse osmosis membranes in several salt solutions. Desalination, 443(out.): $165-171$.

Valentini, L.; Bittolo, S.; Mussolin, L.; Pugno, N. M. 2018. Silkworm silk fibers vs PEEK reinforced rubber luminescent strain gauge and stretchable composites. Composites Science and Technology, 156(mar.): 254-261. 